\title{
Hybrid Skyshine Calculations for Complex Neutron and Gamma-Ray Sources
}

\author{
J. Kenneth Shultis* \\ Kansas State University, Department of Mechanical and Nuclear Engineering \\ Manhattan, Kansas 66506-2503 \\ Received September 14, 1999 \\ Accepted February 15, 2000
}

\begin{abstract}
A two-step hybrid method is described for computationally efficient estimation of neutron and gamma-ray skyshine doses far from a shielded source. First, the energy and angular dependence of radiation escaping into the atmosphere from a source containment is determined by a detailed transport model such as MCNP. Then, an effective point source with this energy and angular dependence is used in the integral line-beam method to transport the radiation through the atmosphere up to $2500 \mathrm{~m}$ from the source. An example spent-fuel storage cask is analyzed with this hybrid method and compared to detailed MCNP skyshine calculations.
\end{abstract}

\section{INTRODUCTION}

Accurate calculation of neutron and gamma-ray skyshine doses is a difficult computational challenge, especially at large distances from the source. To reduce the usually large computational effort of a full transport treatment, several simplified methods have been developed. These include single-scatter techniques, ${ }^{1,2}$ graphical and tabular approaches, ${ }^{3}$ moments-method calculations,${ }^{4} \mathrm{em}$ pirical dose formulas, ${ }^{5-7}$ and methods based on empirical response/Green's functions. ${ }^{8-13}$

Among the latter approaches, the use of line-beam response functions ${ }^{14}$ (LBRFs) has been applied to both gamma-photon and neutron skyshine problems and found to be accurate and computationally efficient. ${ }^{12,13,15} \mathrm{Sev}$ eral computer codes based on this simplified technique are available. ${ }^{10,11,16-20}$ However, this approach requires that knowledge of the energy and directional dependence of the radiation entering the atmosphere first be known. Although the energy and directional spectrum of the actual source is usually known, that of the radiation escaping the shield or source containment is seldom known a priori since the material in and around the source changes the energy and direction of radiation escaping into the atmosphere. Methods based on the LBRF ap-

\footnotetext{
*E-mail: jks@ksu.edu
}

proach can correct only approximately for the energy and angular redistribution caused by a source containment. For example, empirical wall transmission factors ${ }^{10,11}$ or simple exponential attenuation with buildup factors ${ }^{12,17}$ have been used. However, both of these approaches neglect the redirection of radiation as it passes through the containment and, thus, can severely compromise the accuracy of the LBRF approach.

To properly account for the effect of the containment on the skyshine dose, hybrid methods have been developed. In such methods, a transport calculation is performed to obtain the energy and angular distribution of radiation escaping the containment. Then, this escaping radiation is treated as a bare point skyshine source whose resulting skyshine doses can be analyzed with the LBRF method. Previous hybrid analyses have used onedimensional discrete ordinates calculations to determine the energy and angular redistribution of radiation escaping a horizontal slab shield over the radiation source. ${ }^{18,21,22}$ However, most source containments cannot be well modeled by a horizontal slab shield. For a more geometrically complex containment, the use of prescribed angular emission cones or directions has been proposed for the angular redirection of radiation escaping a spent-fuel storage cask. ${ }^{23}$

In this technical note, a new hybrid approach is proposed that uses the MCNP Monte Carlo code ${ }^{24}$ to 
determine the energy and angular distribution of radiation escaping a geometrically complex containment, under the assumption of symmetry about a vertical axis. With the escaping radiation used as an effective point skyshine source, an integral line-beam calculation can then be used to estimate the far-field skyshine dose at distances up to $2500 \mathrm{~m}$ from the skyshine source. For skyshine sources without azimuthal symmetry about the vertical, the proposed approach yields the azimuthally averaged skyshine dose.

\section{THE HYBRID METHOD}

The hybrid method described here is applicable to structures or facilities containing neutron or gamma-ray sources such that the radiation escaping into the atmosphere is azimuthally symmetric about a vertical axis through the source structure. The escaping radiation, however, can have arbitrary polar-angle and energy dependence. Such skyshine radiation sources are typical of spent-fuel storage casks or arrays of containers holding radioactive material. Even for facilities where azimuthal symmetry is not appropriate, this hybrid method can still be used to predict the azimuthally averaged skyshine doses.

In this method, a detailed Monte Carlo transport calculation is first used to determine the energy $E$ and polarangle $\theta$ dependence of the radiation escaping the source containment into the atmosphere. From this calculation, an effective point source of escaping radiation $\hat{S}(E, \theta)$ is estimated. Then, the computationally efficient integral line-beam method is used to transport radiation from this effective source to the distant location at which the skyshine dose is to be calculated.

Implicit in this two-step hybrid scheme is the assumption that the source containment structure has a negligible effect on the transport of radiation through the atmosphere; i.e., once radiation enters the atmosphere, it does not interact again with the source structure. With this assumption, the calculation of the energy and angular dependence of radiation escaping the source containment becomes independent of the subsequent transport of the radiation through the air. In most far-field skyshine calculations, the source and its containment have a negligible effect on the transport of the radiation through the air once the radiation has left the source structure. However, for near-field calculations, this assumption may not be valid.

\section{II.A. Determining the Effective Point Skyshine Source}

The first step in the simplified hybrid skyshine technique is to find the energy and angular distribution of radiation escaping into the atmosphere from the source containment. For almost all nuclear facilities or source containers from which radiation escapes, a near-field ra- diological analysis is almost always performed; i.e., the "contact" dose rates near the exterior surfaces are calculated. The transport of radiation through realistic containments usually requires a detailed transport analysis. Because the geometry of the source containment is usually too complex to be modeled with the simplistic geometries of deterministic transport theory codes, Monte Carlo techniques are usually needed for detailed analyses. Although Monte Carlo codes such as MCNP (Ref. 24) can treat complex material geometries, the high reduction factors designed into most source containments (e.g., spent-fuel storage or shipping casks) require computationally expensive efforts even when sophisticated biasing techniques are used to bias or "draw" particles through the containment structure. Determination of far-field skyshine doses by the same Monte Carlo methods is even more computationally difficult.

The proposed hybrid method requires a transport analysis that can be easily incorporated into a near-field analysis. Since the air exterior to a containment structure is of negligible importance in a near-field analysis, the air can be replaced by a void so that radiation escaping from the structure streams in straight lines from the structure. The soil beneath the air-ground interface is also replaced by a "perfect absorber" to suppress groundshine from contributing to the effective skyshine source. Then, by recording the frequency with which escaping radiation in $G$ energy groups reaches a hemispherical surface (of large radius $R$ centered in the source structure) between a set of $J$ contiguous polar angles (conical annuli), the energy and polar-angle dependence of the escaping radiation can be determined.

Specifically, if on the large-radius hemisphere, $\Phi_{j, g}$ is the fluence of particles in the $g$ 'th energy group $\left(E_{g-1}<\right.$ $\left.E \leq E_{g}\right)$ and with a polar angle in the $j$ 'th interval $\left(\theta_{j-1}<\right.$ $\left.\theta \leq \theta_{j}\right)$, the total $4 \pi$ emission from a bare point isotropic source at the center of the hemisphere that would produce the same observed fluence is

$$
\hat{S}_{j, g}=4 \pi R^{2} \Phi_{j, g}, \quad j=1, \ldots, J, \quad g=1, \ldots, G .
$$

\section{II.A.1. Determining the Skyshine Source with MCNP}

It is relatively easy with the MCNP code to incorporate into a near-field analysis a tally from which the effective point skyshine sources can be calculated directly. One approach is to use an F1 tally (current integrated over a surface) on a sphere centered on the source structure and with a radius much larger than the size of the structure. For such a large tally sphere (e.g., radius of $10^{4} \mathrm{~m}$ ), the spatial variation of where radiation escapes from the structure and begins streaming through the surrounding hemispherical void is negligible so that all particles are essentially normally incident on the tally sphere. Thus, the flow per unit area at any point on the spherical surface is essentially the same as the fluence. Further, by using energy binning (En card) and cosine segmentation 
( $\mathrm{C} n$ card) for the F1 tally, the fluence by energy group and polar-angle interval can be obtained.

The total flow $\mathrm{F} 1_{j, g}$ of particles in the $g$ 'th energy group crossing the spherical tally surface between polar angles $\theta_{j-1}$ and $\theta_{j}$ is related to the fluence $\Phi_{j, g}$ of such particles by

$$
\mathrm{F} 1_{j, g}=A_{j} \Phi_{j, g},
$$

where $A_{j}$ is the area on the tally sphere between polar angles $\theta_{j-1}$ and $\theta_{j}$; namely, $A_{j}=2 \pi R^{2}\left(\omega_{j-1}-\omega_{j}\right)$ with $\omega_{j}=$ $\cos \theta_{j}$, where $\theta_{j}<\theta_{j+1}$ with $\theta_{0}=1$.

Then, combining Eqs. (1) and (2), the emission of the effective point skyshine source for particles in energy group $g$ and polar-angle interval $j$, per actual source particle, is

$$
\hat{S}_{j, g}=f_{j} \mathrm{~F} 1_{j, g}, \quad j=1, \ldots, J, g=1, \ldots, G,
$$

where $f_{j} \equiv 4 \pi R^{2} / A_{j}=2 /\left(\omega_{j-1}-\omega_{j}\right)$, the inverse of the fraction of the total spherical area in the polar-angle interval $j$. One final refinement for the MCNP calculation is to use the tally multiplier CM1 card to multiply the F1 tallies by $f_{j}$ so that the $\mathrm{F} 1$ tally calculates $\hat{S}_{j, g}$ directly, thereby eliminating any postprogram processing. To implement this

\begin{tabular}{|c|c|c|}
\hline & & \\
\hline Fn1:pl & $S_{1}$ & \\
\hline FTn $n$ & $\begin{array}{cc}0 & -1 \\
\end{array}$ & -negative $z$ axis \\
\hline & $\omega_{1}^{\prime} \omega_{2}^{\prime} \omega_{3}^{\prime} \ldots \omega_{J}^{\prime}$ & $\$ J+1$ cosine segment boundaries \\
\hline Mn1 & $f_{1}^{\prime} f_{2}^{\prime} f_{3}^{\prime} \ldots f_{J}^{\prime}$ & $\$$ multiply tally in cosine segment by $4 \pi R^{2} / A_{j}$ \\
\hline & & energy group boundaries $(\mathrm{MeV})$ \\
\hline
\end{tabular}
effective source calculation, the following MCNP input can be used:

where $n$ is the tally number and $p l$ is the source particle type $(\mathrm{N}, \mathrm{P}$ or $\mathrm{N}, \mathrm{P})$. In this implementation, to make the $\omega_{j}^{\prime}$ monotonically increasing, the $\omega_{j}^{\prime}$ are the cosines of $\pi-\theta_{j}$, i.e., the complement of the cosine of the conical polar angle with respect to the upward vertical. Thus, $-1=\omega_{0}^{\prime}<\omega_{1}^{\prime}<\omega_{2}^{\prime}<\ldots<\omega_{J}^{\prime}=0<\omega_{J+1}^{\prime}=1$ and $f_{j}^{\prime}=2 /\left(\omega_{j}^{\prime}-\omega_{j-1}^{\prime}\right)$.

\section{II.B. Determining the Far-Field Skyshine Dose}

The integral line-beam method for skyshine analysis is based on the availability of an $\operatorname{LBRF} \Re(d, E, \phi)$ that gives the dose, per source particle (photon or neutron), at a distance $d$ from a point-source emitting radiation of energy $E$ into an infinite air medium at an angle $\phi$ relative to the source-detector axis. The skyshine dose (or detector response) $R(d)$ arising from a collimated point source that emits $\hat{S}(E, \boldsymbol{\Omega}) d E d \Omega$ particles with energies in $d E$ about $E$ into directions $d \Omega$ about $\boldsymbol{\Omega}$ is found by integrating the LBRF over all source energies and over all emission directions allowed by the source collimation, namely, ${ }^{12,25}$

$$
R(d)=\int_{0}^{\infty} d E \int_{2 \pi} d \Omega \hat{S}(E, \boldsymbol{\Omega}) \Re(d, E, \phi(\boldsymbol{\Omega})) .
$$

The angular integration is over the $2 \pi$ sr subtended by a hemisphere over the source, i.e., over all upward directions. Source radiation directed toward the air-ground interface is neglected.

In the hybrid method, a spherical-polar coordinate system is used with the effective skyshine source at the origin and the polar axis directed vertically upward. Further, the source is assumed to be azimuthally symmetric, to emit radiation into $G$ energy groups, and to be constant within each polar-angle interval. Specifically, in group $g$ and conical interval $j\left(\theta_{j-1} \leq \theta<\theta_{j}\right), \hat{S}_{j, g}$ particles are emitted isotropically with the group midpoint energy $\hat{E}_{g} ; J$ polar-angle intervals are assumed with $0=$ $\theta_{0}<\theta_{1}<\theta_{2}<\ldots<\theta_{J}=\pi / 2$. Thus, the energy and angular dependence of the source can be represented as

$$
S(E, \boldsymbol{\Omega})= \begin{cases}\sum_{g=1}^{G} \frac{\hat{S}_{j, g}}{4 \pi} \delta\left(E-\hat{E}_{g}\right), & \theta_{j-1} \leq \theta \leq \theta_{j} \\ 0, & \text { otherwise } .\end{cases}
$$

With this coordinate system and source approximation, Eq. (4) reduces to

$$
R(d)=\sum_{j=1}^{J} \sum_{g=1}^{G} \frac{\hat{S}_{j, g}}{4 \pi} \int_{0}^{2 \pi} d \psi \int_{\omega_{j-1}}^{\omega_{j}} d \omega \Re\left(d, \hat{E}_{g}, \phi(\omega, \psi)\right)
$$

where $\omega$ is the cosine of the polar angle $\theta$ and the azimuthal angle $\psi$ is defined with respect to the projection of the source-detector axis on the horizontal plane through the source. Here, $\omega_{j-1}=\cos \theta_{j}$ and $\omega_{j}=\cos \theta_{j-1}$ define the permissible range of the cosine of polar angles for particle emission in the $j$ 'th conical interval.

If the skyshine detector is located at an arbitrary vertical distance $h_{d}$ above the source elevation and at a radial distance $x$ from the vertical (polar) axis through the source, the source-to-detector distance $d$ is

$$
d=\sqrt{x^{2}+h_{d}^{2}} .
$$


The emission angle $\phi$ for this geometry is given by ${ }^{12}$

$$
\cos \phi=\sin \theta \cos \psi \cos \zeta+\cos \theta \sin \zeta,
$$

where $\zeta=\tan ^{-1}\left(h_{d} / x\right)$. Then, because of the assumed azimuthal symmetry of the source, Eq. (6) can be written as

$$
\begin{aligned}
R(x)= & \sum_{j=1}^{J} \sum_{g=1}^{G} \frac{\hat{S}_{j, g}}{2 \pi} \\
& \times \int_{\omega_{j-1}}^{\omega_{j}} d \omega\left[\int_{0}^{\pi} d \psi \Re\left(d, \hat{E}_{g}, \phi(\omega, \psi)\right)\right]
\end{aligned}
$$

where $R(x) \equiv R(d)$ is the skyshine dose a horizontal distance $x$ from the source.

Finally, the integrals in Eq. (9) are readily evaluated numerically using standard Gaussian quadrature methods for both integrals. A code for performing the foregoing calculations is available from the World Wide Web. ${ }^{20}$

The foregoing formulation, known as the integral linebeam method, is based on one key approximation, namely, that the ground may be replaced by a continuation of the air medium. For such an infinite air medium, the LBRF $\mathfrak{R}$ depends on only three variables: the source-to-detector distance $x$, the source particle energy $E$, and the angle $\phi$ between the particle emission direction and the sourcedetector axis. The neglect of the air-ground interface has proven to be quite reasonable for most gamma skyshine problems. ${ }^{12,26}$ However, for neutron sources, the ground tends to depress the neutron skyshine dose at large sourcedetector distances compared to the dose in an infinite air medium. At small source-detector distances, soil acts as a reflector compared to an infinite air medium and thus increases the neutron skyshine doses. ${ }^{13,27}$ In LBRF calculations of the neutron skyshine doses, ground correction factors (GCFs) can be incorporated to correct for the air-ground interface., ${ }^{3,13}$ Examples of the air-ground effect are presented later.

\section{II.B.1. Approximation of the LBRF}

An analytical approximation of the LBRF in an infinite air medium is used to evaluate efficiently the integrands in Eq. (9). As originally proposed for the SKYSHINE code ${ }^{9,10}$ and later confirmed by Shultis and Faw ${ }^{15}$; Gui ${ }^{27}$; and Gui, Shultis, and Faw, ${ }^{28}$ the LBRF may be accurately approximated for gamma-ray sources, neutron sources, and secondary gamma-ray skyshine by the following three-parameter function:

$$
\Re(x, E, \phi) \simeq E\left(\rho / \rho_{0}\right)^{2}\left[x\left(\rho / \rho_{0}\right)\right]^{c} e^{a+b x\left(\rho / \rho_{0}\right)},
$$

where $\rho$ is the air density in grams per cubic centimetre and $\rho_{0}$ is the reference air density (equal to $0.0012 \mathrm{~g} / \mathrm{cm}^{3}$ ). The parameters $a, b$, and $c$ depend on the source particle energy $E$, the emission direction $\phi$, and the selected dose unit. Values of these parameters have been determined by fitting Eq. (10) to calculated values of the LBRF for a given source energy and emission direction. Several compilations of the parameters $a, b$, and $c$ are available for photons, neutrons, and secondary photons produced by neutron interactions in the air. ${ }^{14,28,29}$ By using a doubleinterpolation scheme, ${ }^{15,27}$ the approximate LBRF can be made a continuous function of $E$ and $\phi$ as well as $x$.

Earlier compilations ${ }^{10,11}$ of parameters for the neutron and secondary gamma-ray LBRFs used the absorbed air dose to describe the LBRF. For gamma photons, the air-gray is a reasonable approximation for the tissuegray and thus the ambient dose equivalent. However, for neutrons, an air-absorbed dose, in the absence of energy spectral information, cannot be converted into doseequivalent units, which are needed for modern radiological assessments. Recently, Gui used the MCNP code to evaluate the LBRFs for neutrons and the neutron-induced secondary gamma radiation based on three modern doseequivalent units. ${ }^{27}$ From his MCNP-calculated values of the LBRFs, Gui developed a comprehensive tabulation of the fit parameters for use with Eq. (10), and it is this compilation that is used in the neutron calculations presented later. ${ }^{14,27,28}$

The approximate neutron LBRFs (Ref. 14) used by SKYCONES ${ }^{20}$ cover a range of neutron energies from 0.01 to $14 \mathrm{MeV}$ and are generally valid for sourceto-detector distances out to $2500 \mathrm{~m}$. The approximate gamma-ray LBRFs cover a range of photon energies from 0.02 to $100 \mathrm{MeV}$ and are generally accurate for sourceto-detector distances out to $\sim 3000 \mathrm{~m}$ for photon energies $<10 \mathrm{MeV}$. For photon energies between 10 and $100 \mathrm{MeV}$, the maximum range is $\sim 1500 \mathrm{~m}$.

\section{II.B.2. Ground Correction Factors}

An LBRF $\Re(x, E, \phi)$ for an infinite air medium can be corrected for the air-ground interface by a multiplicative GCF (Refs. 3 and 13). In general, the GCF depends on many problem parameters: distance $x$, source energy $E$, emission direction $\boldsymbol{\Omega}(\theta, \psi)$, and elevations of the source and detector above the air-ground interface. However, for conical beams about the vertical and for both the source and detector near (within several metres of) the airground interface, the GCF is a function of only distance $x$, polar angle $\theta$, particle energy $E$, and the neutron dose unit.

Both gamma photons and neutrons are affected by the air-ground interface; however, the effect is generally quite small for gamma rays, and no GCF is needed. Neutron skyshine doses, by contrast, are more severely affected by the air-ground interface, and GCFs are provided by SKYCONES to account for its presence. Examples of skyshine doses with and without an air-ground interface are shown in Figs. 1 and 2 as calculated by SKYCONES and MCNP. Figure 1 shows that the skyshine doses from gamma sources are quite insensitive to the air-ground interface, and no attempt is made by SKYCONES to correct for the interface. However, Fig. 2 shows the 


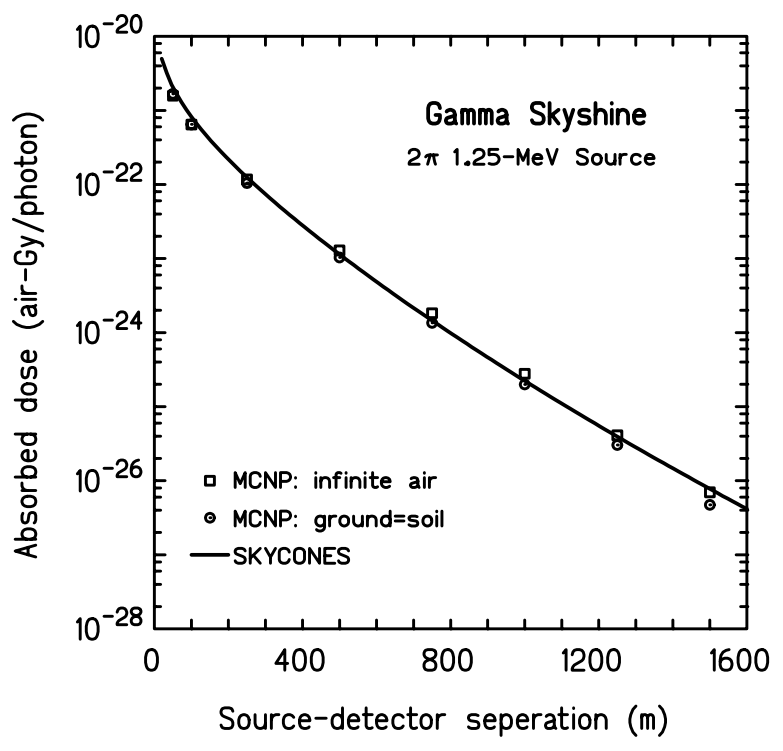

Fig. 1. Gamma skyshine doses with and without an airground interface. The symbols are from the MCNP calculations, and the solid line is from SKYCONES.

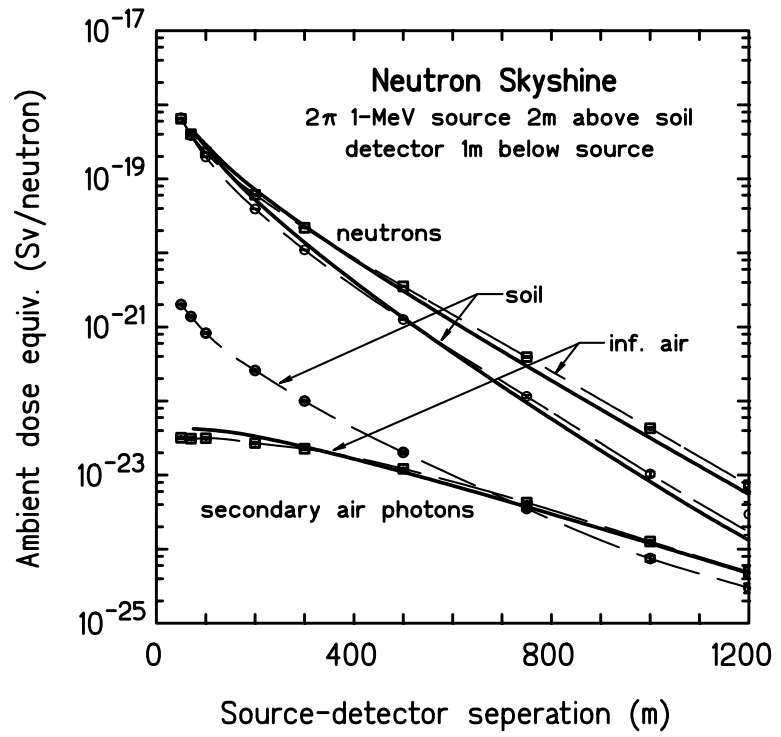

Fig. 2. Neutron skyshine doses with and without an airround interface. The symbols and light dashed lines are from an MCNP calculation, and the solid lines are from SKYCONES

air-ground interface depresses significantly the neutron skyshine dose at large source-detector distances.

In Fig. 2, the SKYCONES-calculated values for the air-ground interface use the optional GCF as devised by Gui. ${ }^{27}$ Gui found from his MCNP calculations that the conical beam GCF for the neutron skyshine dose could be represented by

$$
G C F(x, E, \theta)=a+b x+c x \ln x+d x^{1.5},
$$

where the parameters $a, b, c$, and $d$ depend on the neutron source energy $E$ and the conical half-angle $\theta$.

\section{II.B.3. Secondary-Photon Skyshine Doses for Neutron Sources}

In some neutron skyshine calculations, the skyshine dose arising from secondary photons produced by neutron interactions in the air and ground may be of interest. However, this dose is usually more than an order of magnitude less than the neutron skyshine dose, except at distances $>2000 \mathrm{~m}$, and is usually ignored. For an infinite air medium, the neutron-induced secondary-photon skyshine dose is calculated by SKYCONES based on the approximation of Eq. (10) developed by Gui ${ }^{27}$ for secondary photons. Figure 2 shows that the SKYCONES-calculated secondary-photon dose agrees well with the MCNPcalculated dose in an infinite air medium.

However, unlike the neutron skyshine dose, the secondary-photon skyshine dose depends sensitively on the soil composition when an air-ground interface is considered. Figure 2 shows that the soil can significantly raise the secondary-photon dose at distances near the source, although at levels still well below the primary neutron skyshine dose. At distances far from the source, the soil depresses the secondary-photon dose compared to that in an infinite air medium. At large distances $(>2000 \mathrm{~m})$, the secondary-photon dose may even become greater than the primary neutron skyshine dose. For these extreme cases, the infinite-air secondary-photon dose is conservative (i.e., overpredicts the dose). SKYCONES makes no attempt to correct the secondary-photon skyshine dose for the air-ground interface and calculates only the secondary-photon dose for an infinite air medium, even when optional GCFs are specified for neutron sources.

\section{EXAMPLE CALCULATIONS}

To demonstrate the capabilities of the proposed hybrid skyshine scheme, a spent-fuel storage cask was analyzed by a complete MCNP transport analysis and by the hybrid method. The cask is shown schematically in Fig. 3. Three separate radiation sources were considered:

1. gamma photons from fission/activation products in the spent fuel

2. gamma photons from activation products in the bottom- and top-end fittings

3. neutrons from spontaneous fission of transuranic nuclides and $(\alpha, n)$ reactions.

The storage cask holds 68 fuel assemblies, each of which was assumed to have a $40000 \mathrm{MWd} /$ tonne average burnup with a 10-yr cooling time. The neutron and photon emission rates per assembly are given in Table I. 


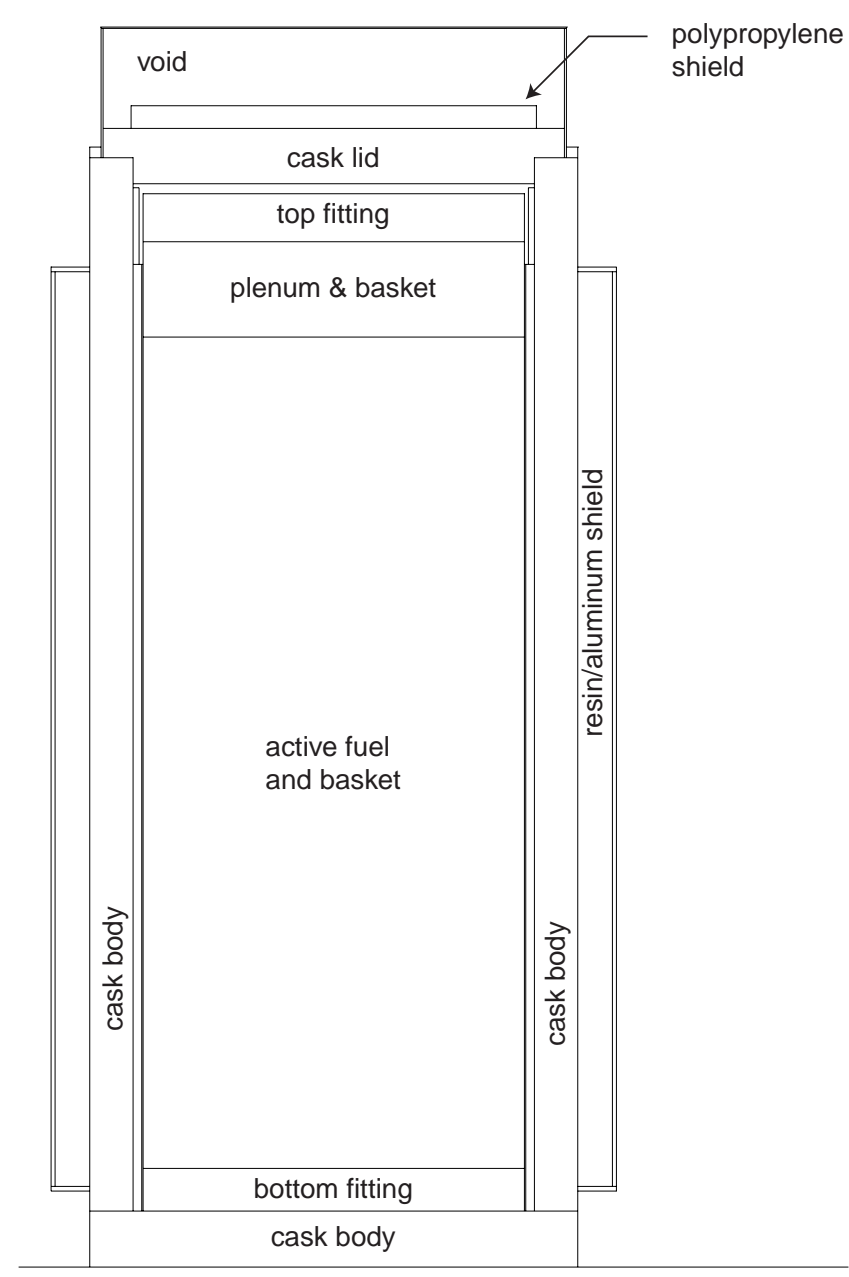

Fig. 3. The spent-fuel storage cask (TN-68) used in the example calculations.

Gamma (neutron) radiation emitted by the spent fuel was consigned to 10 (12) axial zones along the fuel assemblies to account for uneven axial fuel burnup. Further details about source strengths, cask dimensions, and cask composition are reported elsewhere. ${ }^{30}$

\section{III.A. MCNP Skyshine Calculations}

A complete MCNP skyshine simulation of the storage cask resting on an infinite soil-air interface was performed. Symmetry about the cask axis was used and annular tally volumes at various radial distances centered $1 \mathrm{~m}$ above grade recorded radiation doses. Many cylindrical annular cells both inside and outside the cask were given importances that increased with distance from the cask axis to bias particle flow in the outward radial direction. Calculations were performed separately for the spent-fuel neutrons, fuel gamma photons, and activation photons from the end fittings and plenum. For each source, a simulation was performed with infinite air above the cask to obtain the total (direct-plus-skyshine-plusgroundshine) doses, and another simulation was made with the air above the cask replaced by a perfect absorber to obtain only the direct-plus-groundshine dose. The difference between these two calculated doses then yields the skyshine dose.

Results of these calculations are shown in Figs. 4 and 5. As expected, at distances greater than a few hundred metres from the cask, the total dose is dominated by the skyshine component. Close to the cask, however, the direct dose dominates, and the estimated skyshine component, obtained as the difference between two almost equal doses, has relatively large statistical uncertainties.

Because MCNP has been extensively verified from comparisons to results from experiments, analytical solutions, and other transport methods, ${ }^{31-33}$ these MCNPderived skyshine doses for the test cask can then be used as benchmarks to test the capabilities of other simplified methods. Such comparisons are presented as follows for the hybrid method.

\section{TABLE I}

Energy Spectra of Gamma Photons and Neutrons Emitted by the Spent Fuel and by the Fittings/Plenum*

\begin{tabular}{|c|c|c|c|}
\hline $\begin{array}{l}\text { Energy } \\
\text { Range } \\
(\mathrm{MeV})\end{array}$ & $\begin{array}{c}\text { Number of } \\
\text { Particles } \\
\text { (s-1/assembly) }\end{array}$ & $\begin{array}{l}\text { Energy } \\
\text { Range } \\
(\mathrm{MeV})\end{array}$ & $\begin{array}{c}\text { Number of } \\
\text { Particles } \\
\text { (s } s^{-1} / \text { assembly) }\end{array}$ \\
\hline \multicolumn{2}{|c|}{ Spent Fuel (photons) } & \multicolumn{2}{|c|}{ Spent Fuel (neutrons) } \\
\hline 1.33 to 1.66 & $4.826 \times 10^{12}$ & 6.34 to 20.0 & $2.181 \times 10^{6}$ \\
\hline 1.00 to 1.33 & $3.321 \times 10^{13}$ & 3.00 to 6.43 & $2.484 \times 10^{7}$ \\
\hline 0.80 to 1.00 & $3.321 \times 10^{13}$ & 1.85 to 3.00 & $2.756 \times 10^{7}$ \\
\hline 0.60 to 0.80 & $3.321 \times 10^{13}$ & 1.40 to 1.85 & $1.550 \times 10^{7}$ \\
\hline 0.40 to 0.60 & $3.321 \times 10^{13}$ & 0.90 to 1.40 & $2.095 \times 10^{7}$ \\
\hline 0.30 to 0.40 & $3.321 \times 10^{13}$ & 0.40 to 0.90 & $2.283 \times 10^{7}$ \\
\hline 0.20 to 0.30 & $3.321 \times 10^{13}$ & 0.10 to 0.40 & $4.469 \times 10^{6}$ \\
\hline 0.10 to 0.20 & $3.321 \times 10^{13}$ & Total & $1.183 \times 10^{8}$ \\
\hline 0.05 to 0.10 & $3.321 \times 10^{13}$ & Iotal & \\
\hline Total & $1.015 \times 10^{14}$ & & \\
\hline \multicolumn{2}{|c|}{ Top Fitting (photons) } & \multicolumn{2}{|c|}{ Plenum (photons) } \\
\hline 1.33 to 1.66 & $1.246 \times 10^{12}$ & 1.33 to 1.66 & $7.534 \times 10^{11}$ \\
\hline 1.00 to 1.33 & $3.519 \times 10^{11}$ & 1.00 to 1.33 & $2.128 \times 10^{11}$ \\
\hline Total & $1.598 \times 10^{12}$ & Total & $9.662 \times 10^{11}$ \\
\hline \multicolumn{2}{|c|}{ Bottom Fitting (photon) } & & \\
\hline 1.33 to 1.66 & $8.829 \times 10^{11}$ & & \\
\hline 1.00 to 1.33 & $2.493 \times 10^{11}$ & & \\
\hline Total & $1.132 \times 10^{12}$ & & \\
\hline
\end{tabular}

*Source strengths are the number of particles emitted per second per assembly. 


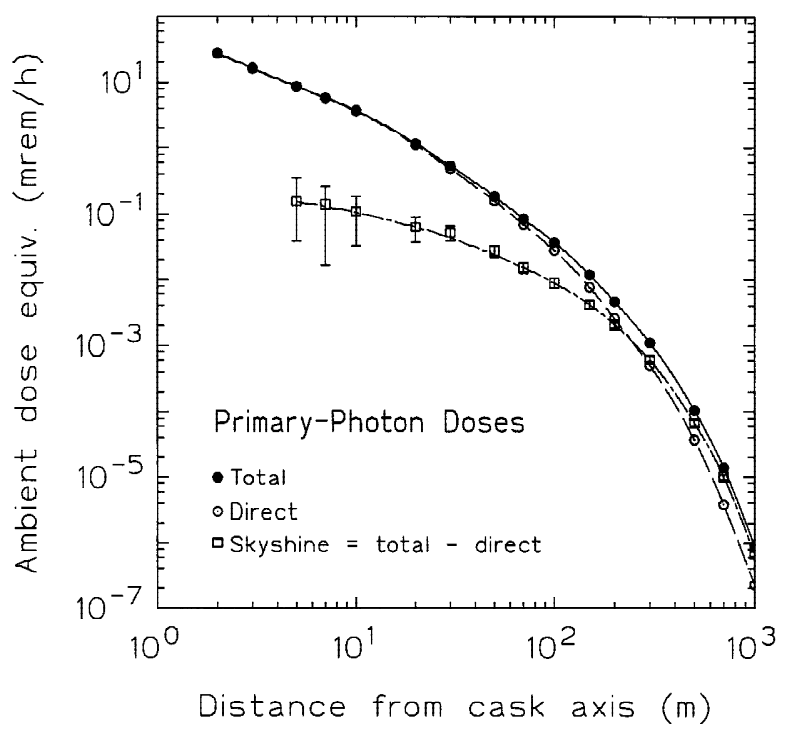

Fig. 4. Results of an MCNP analysis for primary gammaray doses from the TN-68 cask.

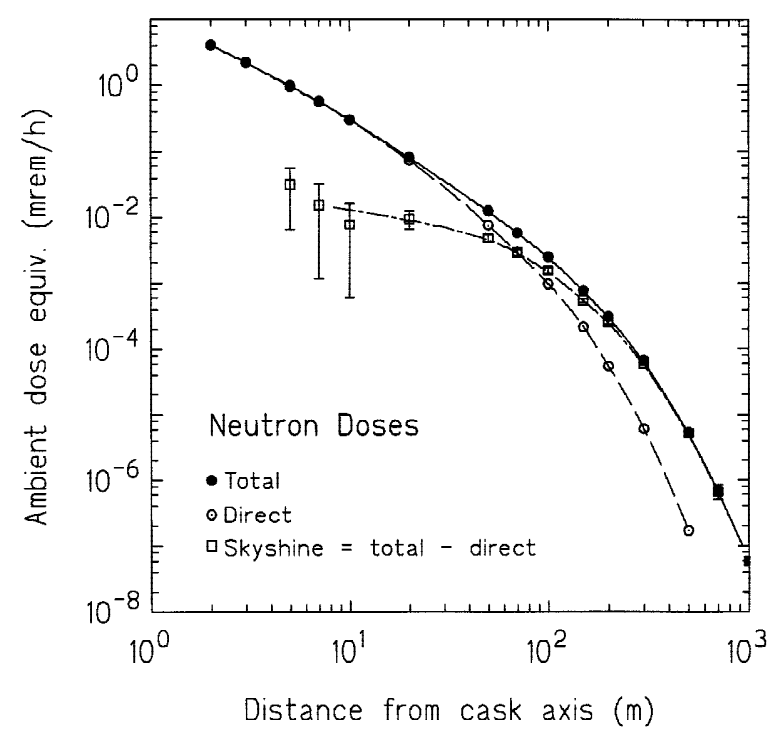

Fig. 5. Results of an MCNP analysis for neutron doses from the TN-68 cask.

\section{III.B. Skyshine Hybrid Source Calculations}

To calculate the energy and polar-angle dependence of the effective skyshine source, the MCNP cask model was placed on the horizontal air-ground interface, and a large spherical tally surface (with a $10^{4}-\mathrm{m}$ radius and centered on the cask axis at the air-ground interface) was placed around the cask. The air was replaced by a void. With the cosine-segmented F1 tally described in Sec. II.A.1, effective skyshine sources $\hat{S}_{j, g}$ for the three primary physical sources were calculated. Nine cosine segments were used between polar angles $0,15,30,45$, $60,70,80,85,88$, and $90 \mathrm{deg}$. To record only the radiation that left the cask in an upward direction, the ground was treated as a perfect absorber to eliminate any groundshine. The F1 tally also produced an energy group tally for the whole sphere (which is that for the upper hemisphere since no particles reached the hemisphere in the ground). Thus, an effective $2 \pi$ source (one conical segment between 0 and $90 \mathrm{deg}$ ), $\hat{S}_{2 \pi, g}$, is also produced by each MCNP simulation.

The energy spectra of the effective $2 \pi$ skyshine sources $\hat{S}_{2 \pi, g}$ along with the physical source spectra in the cask are shown in Figs. 6, 7, and 8. Spectra for each of the nine segmented sources, although similar, do show some segment-to-segment variation. Also shown in Fig. 8 is the effective skyshine source spectrum for secondary photons produced by neutron interactions in the cask material. This secondary-photon source can be treated as a separate skyshine source and should be distinguished from the secondary photons produced by neutrons streaming through the air. In MCNP calculations, separation of these secondary components is usually not attempted, although with the hybrid method, the separate components are easily evaluated.

The polar-angular variation of the different effective skyshine sources, $\sum_{g=1}^{G} \hat{S}_{j, g} / \sum_{g=1}^{G} \hat{S}_{2 \pi, g}$, over all energy groups is shown in Fig. 9. Except for photons from the end fittings and plenum, the other gamma-ray and neutron sources are seen to be preferentially peaked toward large conical angles; i.e., escaping radiation is more likely to leave the cask nearly horizontally than toward the zenith.

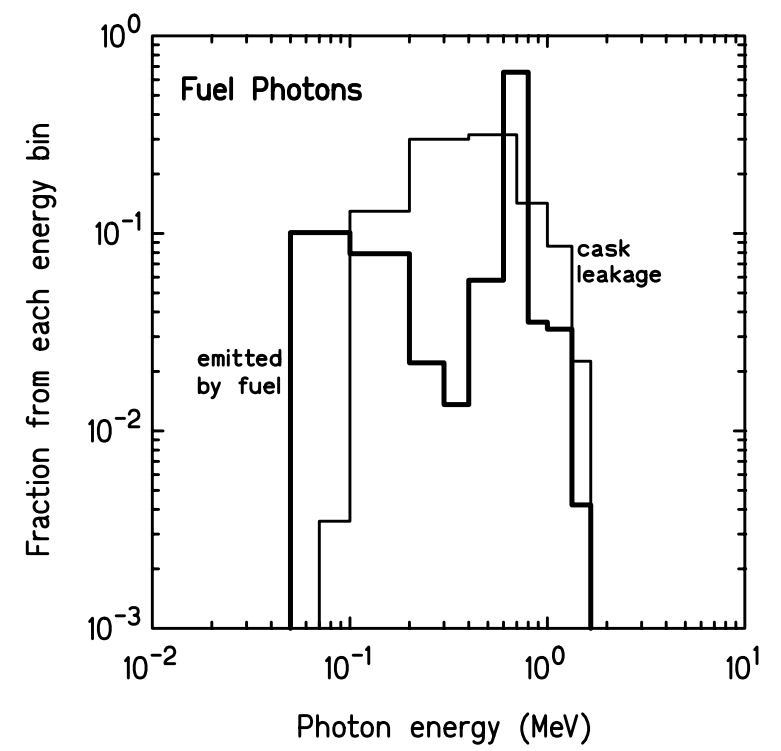

Fig. 6. The $2 \pi$ energy spectrum of gamma photons emitted by the fuel (heavy line) and those that leak through the cask into the atmosphere (light line). 


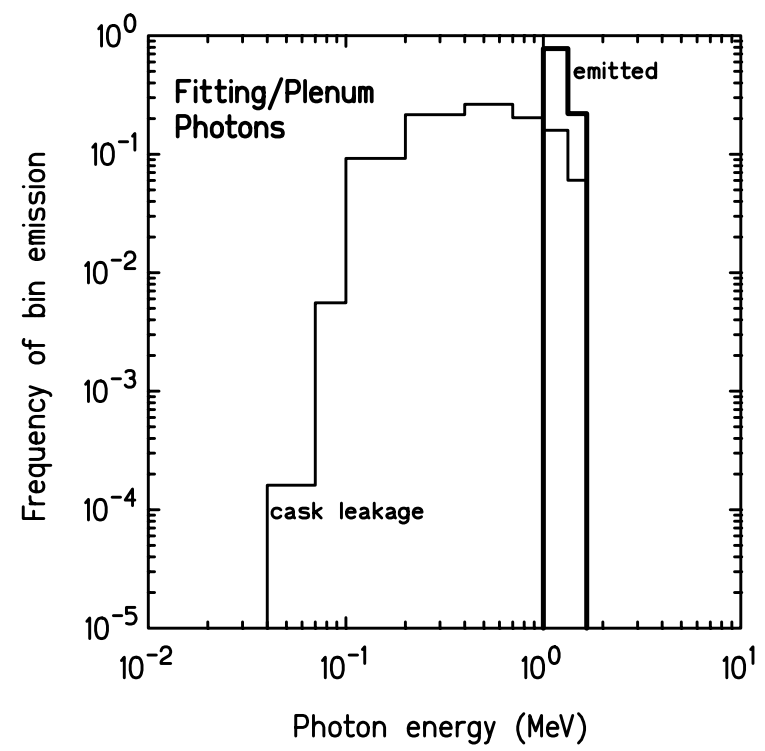

Fig. 7. The $2 \pi$ energy spectrum of gamma photons emitted by activation products in the end fittings (heavy line) and those that subsequently leak through the cask into the atmosphere (light line).

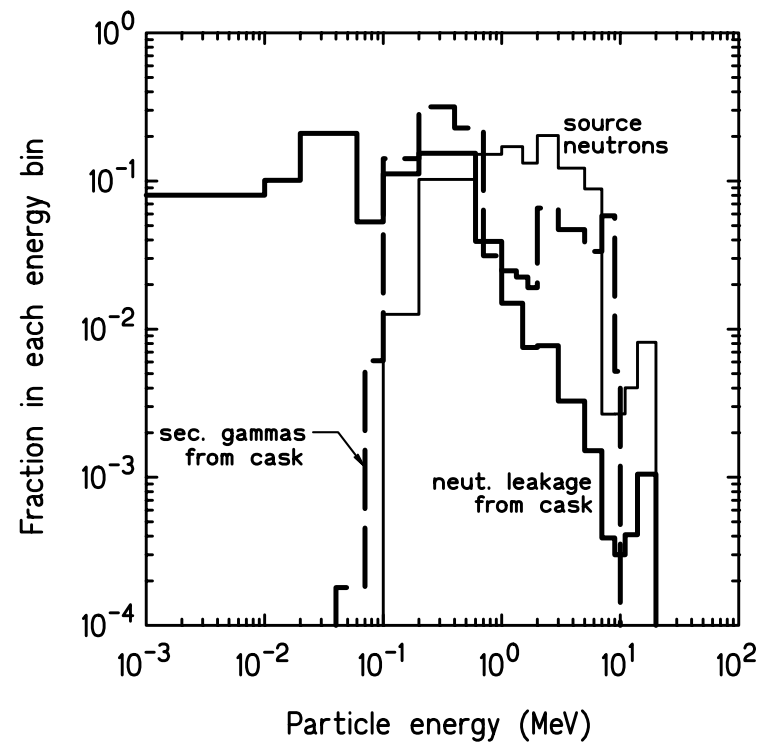

Fig. 8. The $2 \pi$ energy spectrum of neutrons emitted by the fuel (light line) and those that subsequently leak through the cask into the atmosphere (heavy line). The dashed line shows the energy spectrum of secondary photons created by neutron interactions in the cask and that subsequently escape into the atmosphere.

\section{III.C. Hybrid Skyshine Calculations}

With the effective skyshine source strengths calculated by MCNP, the integral line-beam method was used to calculate the resulting skyshine doses at large dis-

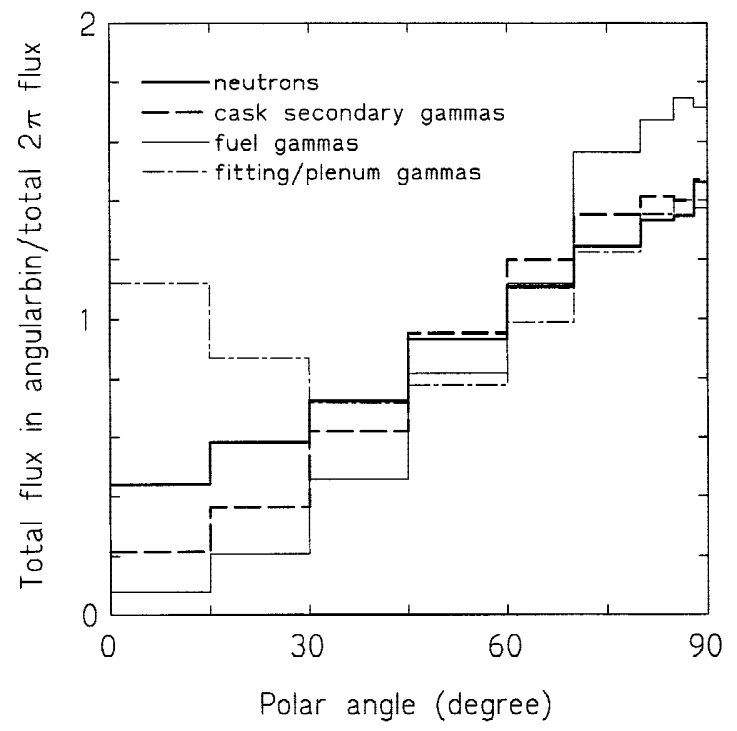

Fig. 9. The angular distribution of radiation escaping from the cask. The quantity $\sum_{g=1}^{G} \hat{S}_{j, g} / \sum_{g=1}^{G} \hat{S}_{2 \pi, g}$ is plotted against the polar-angle bin. An isotropic source would have a constant value of unity.

tances from the cask. This LBRF calculation was performed with the SKYCONES code ${ }^{20}$ which can treat a set of multigroup, conically segmented, point, isotropic, neutron, or photon sources. This code estimates the skyshine doses for each conical segment and can sum the results for each segment.

Comparison of the hybrid results using nine conical segments and the MCNP results are shown in Fig. 10 for

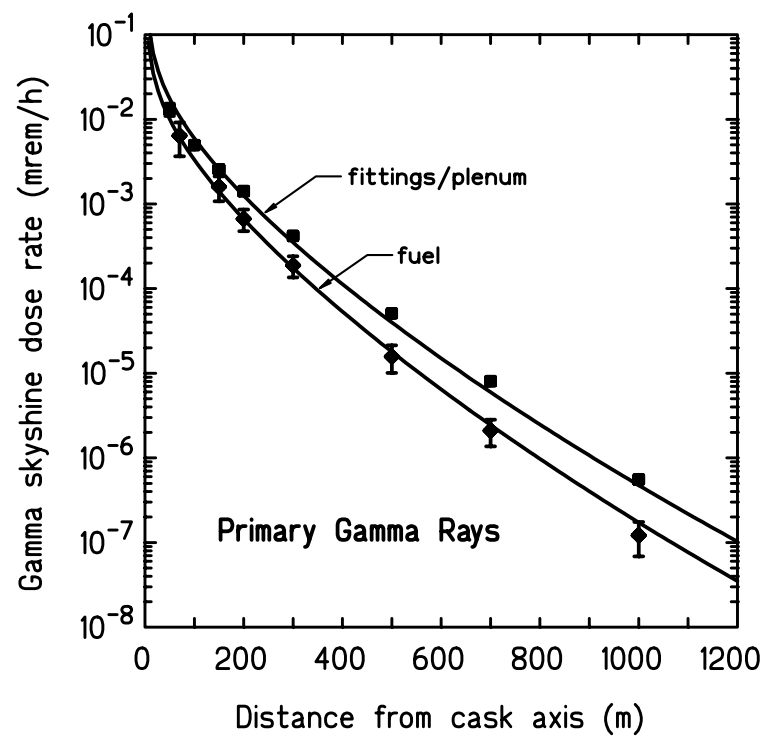

Fig. 10. Comparison of the primary gamma-ray skyshine dose as calculated by MCNP and the hybrid method using SKYCONES and nine conical segments. Results are shown separately for the fuel photons and the end fittings/plenum photons. 
the two primary gamma-photon sources and in Fig. 11 for the neutron source. The agreement between the two methods is seen to be remarkably good. In Fig. 11, the use of a GCF for the neutron skyshine dose produces only a small change compared to the dose without the GCF, but it is a change that does give better agreement with the MCNP results. Also shown in Fig. 11 are the skyshine doses (estimated by the hybrid method) produced from the secondary photons produced in the cask and in the air. Although the cask secondary-photon dose is insensitive to the soil being replaced by a continuation of the air in the LBRF method, the air secondary-photon dose depends sensitively on the soil composition (see Fig. 2).

Finally, in Fig. 12, the effect is shown of using nine polar segments and a single $2 \pi$ segment. For the neutron skyshine dose, both approximations give nearly the same result. However, the strong directional anisotropy of the fuel gamma photons requires a nine-segment hybrid analysis to produce good agreement with the MCNP results.

\section{III.D. Calculational Considerations}

The detailed MCNP far-field skyshine calculations for the foregoing spent-fuel storage cask example required $\sim 7500$ min of computing time on a $200-\mathrm{MHz}$ personal computer. This time does not include the considerable MCNP calculations needed to develop the far-field models and the proper biasing needed to improve the scoring at distances up to $1000 \mathrm{~m}$ from the cask. Details of the MCNP models and the skyshine calculations are available. ${ }^{30}$ By contrast, the hybrid method required $3700 \mathrm{~min}$

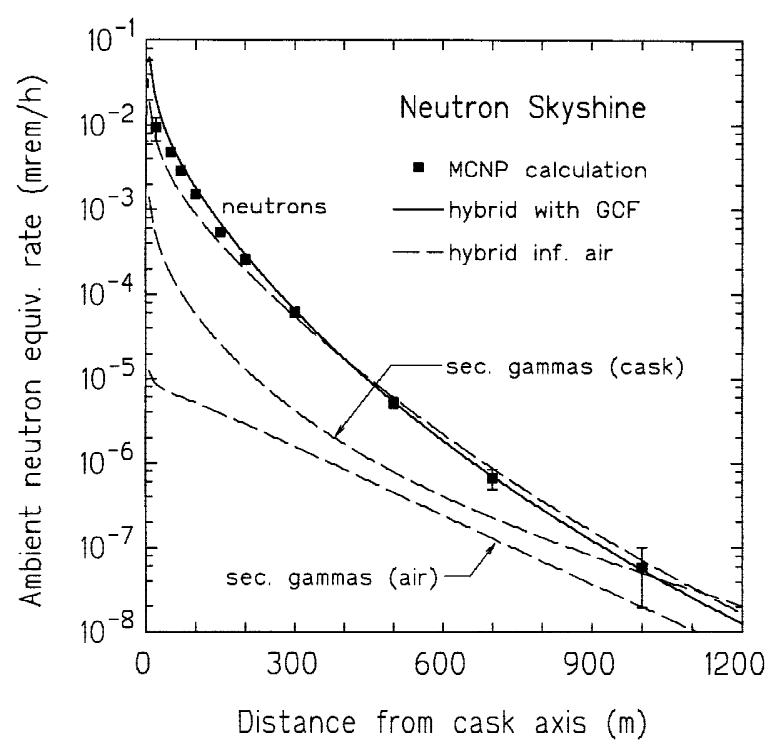

Fig. 11. Comparison of the neutron skyshine dose as calculated by MCNP and the hybrid method using SKYCONES. A single $2 \pi$ hemisphere (one conical segment) was used. Skyshine doses for secondary photons produced in the cask and air are shown by the two lower dashed lines.

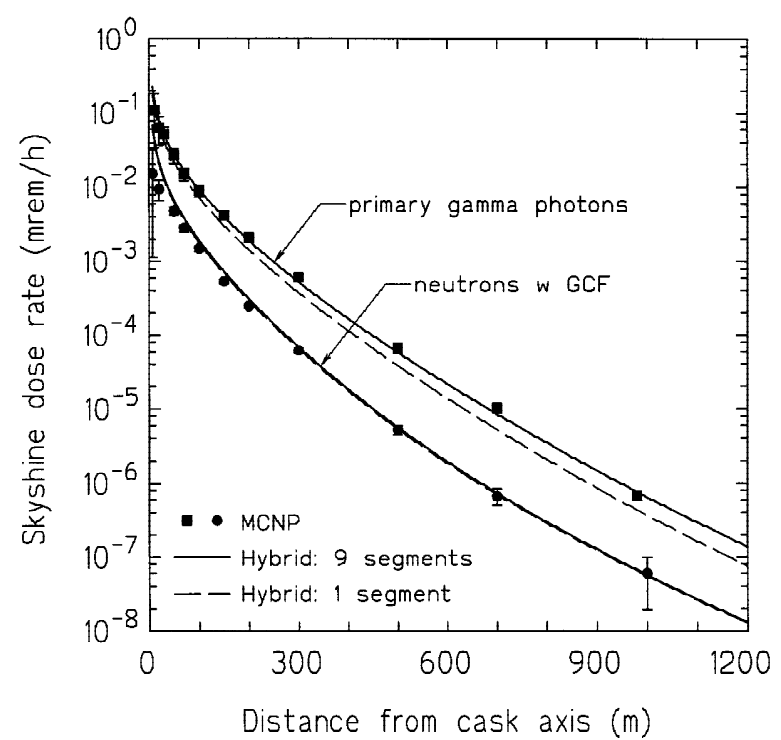

Fig. 12. Hybrid calculations of primary gamma-ray and neutron skyshine doses using nine conical segments (heavy solid lines) and using a single $2 \pi$ hemisphere (dashed lines).

to obtain the effective skyshine source strengths, while the SKYCONES calculations of the far-field skyshine doses required $<1 \mathrm{~min}$. Of course, if a near-field MCNP calculation is to be performed to obtain doses near the source structure, the determination of effective skyshine source strengths can be incorporated into such an analysis with little increase in computational effort. In this case, the far-field skyshine doses by the hybrid method can be obtained with negligible computational expense.

Although the foregoing example is for a single cask, an array of casks could be treated similarly by computing an effective point skyshine source for the array. However, it seldom is necessary to do so, and it is conservative to estimate the far-field skyshine dose from an array of casks as the sum of the skyshine doses produced by each cask individually. This is because radiation leaving a cask and contributing to the far-field skyshine dose usually is emitting in an upward direction and does not first interact with neighboring casks. Only for very closely packed arrays or arrays in which significant subcritical fission multiplication occurs is it necessary to consider caskcask interactions.

\section{CONCLUSIONS}

A two-step hybrid method that uses MCNP to calculate the energy and angular dependence of radiation escaping a complex source containment and the integral line-beam method for subsequent skyshine calculations has been proposed and tested on a realistic skyshine problem. The agreement between the hybrid method and a 
full MCNP skyshine analysis is excellent. Moreover, the hybrid method requires only a small fraction of the computational expense needed for a complete MCNP skyshine analysis.

\section{ACKNOWLEDGMENTS}

The author wishes to thank Transnuclear, Inc., and Northern State Power Company, who sponsored skyshine calculations that were later used in the development of the hybrid scheme.

\section{REFERENCES}

1. D. K. TRUBEY, "The Single-Scattering Approximation to the Solution of the Gamma-Ray Air-Scattering Problem," Nucl. Sci. Eng., 10, 102 (1961).

2. C. M. KITAZUME, "A Simple Calculation for Air-Scattered Gamma-Rays,” J. Nucl. Sci. Technol., 5, 464 (1968).

3. A. ARONSON and R. L. FRENCH, "Air, Ground, and Structure Scattering of Radiation," Reactor Handbook, "Shielding," Vol. III, Part B, 2nd ed., E. P. BLIZARD and L. S. ABBOTT, Eds., Wiley-Interscience, New York (1962).

4. G. L. SIMMONS and C. M. EISENHAUER, "Neutron Dose and Fluence Distributions in an Infinite Air Medium," Technical Note 745, U.S. National Bureau of Standards (1973).

5. K. HAYASHI and T. NAKAMURA, "Analytical Dose Evaluation of Neutron and Secondary Gamma-Ray Skyshine from Nuclear Facilities," Nucl. Sci. Eng., 91, 332 (1985).

6. G. R. STEVENSON and R. H. THOMAS, "A Simple Procedure for the Estimation of Neutron Skyshine from Proton Accelerators," Health Phys., 46, 1, 115 (1984).

7. G. B. STAPLETON, K. O'BRIEN, and R. H. THOMAS, "Accelerator Skyshine: Tyger, Tyger Burning Bright," presented at 37th Annual Mtg. Health Physics Society, Columbus, Ohio, 1992.

8. R. G. ALSMILLER, Jr., J. BARISH, and R.C. CHILDS, "Skyshine at Neutron Energies $\leq 400 \mathrm{MeV}$," Part. Accel., 11, 131 (1981).

9. J. H. PRICE, D. G. COLLINS, and M. B. WELLS, "Utilization Instructions for SKYSHINE," RRA-N7608, Radiation Research Associates (1976).

10. C. M. LAMPLEY, "The SKYSHINE-II Procedure: Calculation of the Effects of Structure Design on Neutron, Primary Gamma-Ray, and Secondary Gamma-Ray Dose Rates in Air," RRA-T7901 (NUREG/CR-0791), Radiation Research Associates (1979).

11. C. M. LAMPLEY, M. C. ANDREWS, and M. B. WELLS, "The SKYSHINE-III Procedure: Calculation of the Effects of
Structure Design on Neutron, Primary Gamma-Ray and Secondary Gamma-Ray Dose Rates in Air," RRA T8209A, (RSIC Code Collection CCC-289), Radiation Research Associates (1988).

12. J. K. SHULTIS, R. E. FAW, and M. S. BASSETT, "The Integral Line-Beam Method for Gamma Skyshine Analysis," Nucl. Sci. Eng., 107, 228 (1991).

13. A. A. GUI, J. K. SHULTIS, and R. E. FAW, "Neutron Skyshine Calculations with The Integral Line-Beam Method," Nucl. Sci. Eng., 127, 230 (1997).

14. J. K. SHULTIS and R. E. FAW, "Response Functions for Neutron and Gamma-Ray Skyshine Analysis," 271, Engineering Experiment Station, Kansas State University (1995); see also DLC-188/SKYDATA-KSU, Radiation Shielding Information Center, Oak Ridge National Laboratory; also available on the Internet (www.mne.ksu.edu/ jks).

15. J. K. SHULTIS and R. E. FAW, "Extensions to the Integral Line-Beam Method for Gamma-Ray Skyshine Analyses," SAND94-2019, Sandia National Laboratories (1995).

16. "MicroSkyshine User's Manual," Grove Engineering (1987).

17. J. K. SHULTIS, R. E. FAW, and R. C. BROCKHOFF, "SKYDOSE: A Code for Gamma Skyshine Calculations Using the Integral Line-Beam Method," SAND95-1748, Sandia National Laboratories (Aug. 1995); see also CCC-646/ SKYSHINE-KSUCCC, Radiation Shielding Information Center, Oak Ridge National Laboratory; also available on the Internet (www.mne.ksu.edu/ jks).

18. J. K. SHULTIS, R. E. FAW, and M. H. STEDRY, "McSKY: A Hybrid Monte-Carlo Line-Beam Code for Shielded Gamma Skyshine Calculations," SAND95-1747, Sandia National Laboratories (Aug. 1995); see also CCC-646/SKYSHINEKSUCCC, Radiation Shielding Information Center, Oak Ridge National Laboratory; also available on the Internet (www. mne.ksu.edu/ jks).

19. J. K. SHULTIS, R. E. FAW, and F. A. KHAN, "SKYNEUT: A Code for Neutron Skyshine Calculations Using the Integral Line-Beam Method," Report 9503, Institute for Computational Research in Engineering and Science, Kansas State University (June 1995); see also CCC-646/SKYSHINEKSUCCC, Radiation Shielding Information Center, Oak Ridge National Laboratory; also available on the Internet (www. mne.ksu.edu/ jks).

20. J. K. SHULTIS, "SKYCONES: A Code for Neutron and Photon Skyshine Calculations from Annular Conical Sources," Report 9903, Institute for Computational Research in Engineering and Science, Kansas State University (June 1995); also available on the Internet (www.mne.ksu.edu/ jks).

21. B. KECK and P. HERCHENRODER, "Nachrechneneines Gamma-Skyshine-Benchmark Experiments," Atomwirtschaft (1982). 
22. T. GIANAKON, "Calculation of Neutron Line-Beam Response Function with TWODANT," MS Thesis, Kansas State University, Department of Nuclear Engineering (1989).

23. N. E. HERTEL, D. G. NAPOLITANO, and M. W. GRANUS, "Benchmarking and Application of SKYSHINEIII on Dry Storage Casks," Proc. Radiation Protection and Shielding Division Topical Conference, Vol. II, 59-68, American Nuclear Society, La Grange Park, Illinois, April, 1998.

24. "MCNP-A General Monte Carlo N-Particle Transport Code," Version 4b, LA-12625-M, J. F. BRIESMEISTER, Ed., Los Alamos National Laboratory (1997).

25. J. K. SHULTIS and R. E. FAW, Radiation Shielding, American Nuclear Society, La Grange Park, Illinois (2000).

26. F. A. KAHN, "Gamma-Ray Skyshine LBRF Near an AirGround Interface,” MS Thesis, Kansas State University (1995).

27. A. A. GUI, "Response Functions for Neutron Skyshine Analyses," PhD Dissertation, Kansas State University (1994).
28. A. A. GUI, J. K. SHULTIS, and R. E. FAW, "Response Functions for Neutron Skyshine Analyses," Nucl. Sci. Eng., 125, 111 (1997).

29. R. C. BROCKHOFF, J. K. SHULTIS, and R. E. FAW, "Skyshine Line-Beam Response Functions for 20- to 100-MeV Photons," Nucl. Sci. Eng., 123, 282 (1996).

30. J. K. SHULTIS, "Radiation Analysis of a Spent-Fuel Storage Cask," Report 290, Engineering Experiment Station, Kansas State University (2000).

31. D. J. WHALEN, D. A. CARDON, J. L. UHLE, and J. S. HENDRICKS, "MCNP: Neutron Benchmark Problems," LA12212, Los Alamos National Laboratory (1991).

32. D. J. WHALEN, D. E. HOLLOWELL, and J. S. HENDRICKS, "MCNP: Photon Benchmark Problems," LA-12196, Los Alamos National Laboratory (1991).

33. R. H. OLSHER, H.-.H. HSU, and W. F. HARVEY, "Benchmarking the MCNP Monte Carlo Code with a Photon Skyshine Experiment," Nucl. Sci. Eng., 114, 219 (1993). 